\title{
TOPOLOGICAL INVARIANTS OF A FAN ASSOCIATED TO A TORIC VARIETY
}

\author{
TIMOTHY J. FORD
}

\begin{abstract}
Associated to a toric variety $X$ of dimension $r$ over a field $k$ is a fan $\Delta$ on $\mathbb{R}^{r}$. The fan $\Delta$ is a finite set of cones which are in one-to-one correspondence with the orbits of the torus action on $X$. The fan $\Delta$ inherits the Zariski topology from $X$. In this article some cohomological invariants of $X$ are studied in terms of whether or not they depend only on $\Delta$ and not $k$. Secondly some numerical invariants of $X$ are studied in terms of whether or not they are topological invariants of the fan $\Delta$. That is, whether or not they depend only on the finite topological space defined on $\Delta$. The invariants with which we are mostly concerned are the class group of Weil divisors, the Picard group, the Brauer group and the dimensions of the torsion free part of the étale cohomology groups with coefficients in the sheaf of units. The notion of an open neighborhood of a fan is introduced and examples are given for which the above invariants are sufficiently fine to give nontrivial stratifications of an open neighborhood of a fan all of whose maximal cones are nonsimplicial.
\end{abstract}

\section{INTRODUCTION}

Let $k$ be a field. Let $N=\mathbb{Z}^{r}$ and denote by $T_{N}$ the $k$-torus on $N$. Let $\Delta$ be a finite fan on $N \otimes \mathbb{R}$ and $X=T_{N} \operatorname{emb}(\Delta, k)$ the toric variety over $k$ associated to $\Delta$ [2], [6], [10]. This defines a functor $T_{N}$ emb on the product category

$$
\begin{array}{ccc}
\text { (finite fans on } N \otimes \mathbb{R}) \times(\text { fields }) & \stackrel{T_{N} \text { emb }}{\longrightarrow} & \text { (toric varieties) } \\
(\Delta, k) & \mapsto & T_{N} \operatorname{emb}(\Delta, k)
\end{array} .
$$

We define the topology on $\Delta$ as follows (cf. [4, pp. 137-138]). The orbit space $\tilde{X}$ of $X$ under the action of the torus $T_{N}$ is in one-to-one correspondence with the finite set of cones that belong to $\Delta$. There is a topology on $\tilde{X}$ inherited from $X$ by the continuous function $X \rightarrow \tilde{X}$. Identifying a cone $\sigma \in \Delta$ with the orbit $\operatorname{orb} \sigma$ in $\tilde{X}$, we see that the topology on $\tilde{X}$ corresponds to the topology on $\Delta$ under which the open sets are the subfans of $\Delta$. The fan $\Delta$ is now a two-faced beast. On the one hand $\Delta$ is an object in the category of fans on $N \otimes \mathbb{R}$. At the same time $\Delta$ is an object of the category of finite topological spaces. To distinguish between these roles played by $\Delta$, we denote by $\Delta_{\text {fan }}$ the object in the category of fans on $N \otimes \mathbb{R}$ and by $\Delta_{\text {top }}$ the object in the category of finite topological spaces. This defines a functor $\mathfrak{T}$ (which factors via $T_{N}$ emb through the category of toric varieties)

$$
\begin{array}{ccc}
\text { (finite fans on } N \otimes \mathbb{R}) \times \text { (fields) } & \stackrel{\mathfrak{T}}{\rightarrow} & \text { (finite top. spaces) } \\
\left(\Delta_{\text {fan }}, k\right) & \mapsto & \Delta_{\text {top }}
\end{array} .
$$

In Section 2 we consider some invariants of $\Delta_{\text {fan }}$ that are constant for all $k$. Suppose $\gamma\left(\Delta_{f a n}, k\right)$ is an invariant that is defined for any pair $\left(\Delta_{f a n}, k\right)$ (in this article $\gamma$ is usually an abelian group). We call $\gamma$ a fan invariant in case $\gamma\left(\Delta_{\text {fan }}, k\right)$ depends only on $\Delta_{\text {fan }}$ and

1991 Mathematics Subject Classification. 14M25, secondary 13A20, 16A16, 14F20. 
not on $k$ - that is, given a fan $\Delta_{f a n}, \gamma\left(\Delta_{f a n}, k_{1}\right) \cong \gamma\left(\Delta_{f a n}, k_{2}\right)$ for every pair of fields $k_{1}$, $k_{2}$. We show that the Brauer group $\mathrm{B}()$ is not a fan invariant for nonsingular fans. This is an observation based on a theorem of Hoobler and [3, Theorem 1.1]. In [3, Theorem 1.1] a complete computation of the Brauer group of a nonsingular toric variety $X=T_{N} \operatorname{emb}(\Delta)$ over an algebraically closed field $k$ was given in terms of the so-called invariant factors of the fan $\Delta$. In Theorem 2.3 we give the Brauer group of $T_{N} \operatorname{emb}(\Delta, k)$ for any field $k$ in terms of the Brauer group and Galois group of $k$. The main result of Section 2 is Theorem 2.5 in which it is stated that the class group, $\mathrm{Cl}($ ), the Picard group, Pic( ), and the relative cohomology group, $H^{2}\left(K() / X()\right.$ ét, $\mathbb{G}_{m}$ ) (where $K(X)$ is the function field of $X$ ), are fan invariants. The proof of Theorem 2.5 follows from that of [⿴囗十

In Section 3 we consider some invariants of $\Delta_{\text {fan }}$ that are constant on fibers of the map $\mathfrak{T}$ in (2), hence depend only on $\Delta_{\text {top }}$. That is, suppose we have an invariant $\beta\left(\Delta_{\text {fan }}\right)$ (usually a numerical invariant) associated to any fan $\Delta_{\text {fan }}$. If two fans $\Delta_{1}, \Delta_{2}$ have the same $\beta$ invariant whenever $\left(\Delta_{1}\right)_{\text {top }} \cong\left(\Delta_{2}\right)_{\text {top }}$, then we say $\beta$ is a topological invariant of $\Delta_{\text {fan }}$. We consider several invariants, all being cohomologically defined. The first sequence is defined by

$$
\rho_{0}=\operatorname{dim}_{\mathbb{Q}}\left[H^{0}\left(X_{\text {ét }}, \mathbb{G}_{m}\right) / k^{*} \otimes \mathbb{Q}\right],
$$

and for $i \geq 1$,

$$
\rho_{i}=\operatorname{dim}_{\mathbb{Q}}\left[H^{i}\left(X_{\text {ét }}, \mathbb{G}_{m}\right) \otimes \mathbb{Q}\right]
$$

Also set

$$
\rho_{1}^{\prime}=\operatorname{dim}_{\mathbb{Q}}[\mathrm{Cl}(X) \otimes \mathbb{Q}] .
$$

For $0 \leq i \leq 2$ these numbers are finite and are fan invariants. The first main result of Section 3 lists some facts about $\rho_{0}$ and $\rho_{1}^{\prime}$.

Theorem 3.1. Let $N=\mathbb{Z}^{r}, \Delta$ a fan on $N \otimes \mathbb{R}, X=T_{N} \operatorname{emb}(\Delta)$, and $s=\operatorname{dim}_{\mathbb{R}} \mathbb{R}\left|\Delta_{\text {fan }}\right|$ (that is, s is the dimension of the $\mathbb{R}$-vector space spanned by the vectors in the support $\left.\left|\Delta_{\text {fan }}\right|\right)$. Then

(a) $\rho_{0}=r-s$, hence is a fan invariant, but not a topological invariant.

(b) Suppose $\Delta_{\text {fan }}$ contains a cone $\sigma$ such that $\operatorname{dim} \sigma=r$. This is true for example if $\Delta_{\text {fan }}$ is a complete fan on $N \otimes \mathbb{R}$. Then $\rho_{0}=0$ and $\rho_{0}$ is a topological invariant of $\Delta_{\text {fan }}$.

(c) $\rho_{1}^{\prime}=\operatorname{dim}_{\mathbb{Q}}(\mathrm{Cl}(X) \otimes \mathbb{Q})=\#(\Delta(1))-s$. If $\operatorname{dim} \Delta_{\text {top }}=r$, then $\rho_{1}^{\prime}$ is a topological invariant of $\Delta_{\text {fan }}$.

(d) The number $\rho_{0}-\rho_{1}^{\prime}$ is a topological invariant of $\Delta_{\text {fan }}$.

The second main result of Section 3 gives some results on $\rho_{0}, \rho_{1}$ and $\rho_{2}$ for simplicial fans.

Theorem 3.2. Let $N=\mathbb{Z}^{r}$. Let $\Delta$ be a simplicial fan on $N \otimes \mathbb{R}$ and $s=\operatorname{dim}_{\mathbb{R}} \mathbb{R}\left|\Delta_{\text {fan }}\right|$. Then

(a) $\rho_{1}=\#(\Delta(1))-s$.

(b) $\rho_{2}=0$ hence is a topological invariant of $\Delta_{\text {fan }}$.

(c) If $\operatorname{dim} \Delta_{\text {top }}=r$, then $\rho_{1}$ is a topological invariant of $\Delta_{\text {fan }}$.

(d) $\rho_{0}-\rho_{1}+\rho_{2}$ is a topological invariant of $\Delta_{\text {fan }}$.

The third main result of Section 3 gives some results on $\rho_{0}, \rho_{1}$ and $\rho_{2}$ for 3-dimensional fans. 
Theorem 3.5. Let $\Delta$ be a fan on $N \otimes \mathbb{R}$. Let $\sigma_{0}, \ldots, \sigma_{w}$ be the maximal cones in $\Delta$. Assume $\sigma_{i} \cap \sigma_{j}$ is simplicial for each $i \neq j$. These assumptions are satisfied for example if $\operatorname{dim} \Delta_{\text {top }} \leq 3$. Then

(a)

$$
\rho_{1}+s+\sum_{i=0}^{w}\left(\#\left(\Delta\left(\sigma_{i}\right)(1)\right)-s_{i}\right)=\rho_{2}+\#(\Delta(1)),
$$

where we set $s_{i}=\operatorname{dim} \sigma_{i}$ for each $i=0, \ldots, w$ and $s=\operatorname{dim}_{\mathbb{R}} \mathbb{R}\left|\Delta_{\text {fan }}\right|$.

(b) $\rho_{0}-\rho_{1}+\rho_{2}$ is a topological invariant of $\Delta_{\text {fan }}$.

In Section 1 we introduce the notion of an open neighborhood $B$ of a fan $\Delta$. This is a subset of the fiber $\mathfrak{T}^{-1}\left(\Delta_{\text {top }}\right)$ that is parametrized by a dense subset of a real manifold. Let $\mathcal{S F}$ denote the sheaf of $\Delta$-linear support functions on the topological space $\Delta_{t o p}$. It was shown in [4] that the numbers $\rho_{i}, 1 \leq i \leq 2$, can be determined by the cohomology of the sheaf $\mathcal{S F}$ on the finite topological space $\Delta_{t o p}$. Therefore we define another sequence of invariants by

$$
\kappa_{i}=\operatorname{dim}_{\mathbb{Q}}\left[H^{i}\left(\Delta_{t o p}, \mathcal{S F}\right) \otimes \mathbb{Q}\right]
$$

for $i \geq 0$. We consider the stratification of $B$ by the numerical invariant $\kappa_{0}$. Several examples are given for which the stratification of $B$ is nontrivial. We conjecture that $\kappa_{0}=3$ on a nonempty open subset of $B$ if $\Delta$ is a complete fan on $\mathbb{R}^{3}$ such that every maximal cone of $\Delta$ is nonsimplicial. Algorithm 4.4 is presented which computes an upper bound for $\kappa_{0}$. For complete 3-dimensional fans, this algorithm can be used to compute an upper bound for $\rho_{1}$ and $\rho_{2}$.

For the benefit of the reader the following notation will be fixed throughout the rest of the paper.

\begin{tabular}{|ll|ll|}
\hline$k$ & a field & $r$ & a positive integer \\
$N$ & $=\mathbb{Z}^{r}$ & $M$ & $=\operatorname{Hom}_{\mathbb{Z}}(N, \mathbb{Z})$ \\
$\Delta$ & a finite rational fan on $N \otimes \mathbb{R}$ & $X$ & $=T_{N} \operatorname{emb}(\Delta, k)$ toric variety \\
$\Delta_{\text {fan }}$ & object in the category of fans & $\Delta_{\text {top }}$ & finite topological space \\
$\left|\Delta_{\text {fan }}\right|$ & support of the fan $\Delta$ & functor that maps $\left(\Delta_{\text {fan }}, k\right)$ to $\Delta_{\text {top }}$ \\
$\mathrm{Cl}(X)$ & $\begin{array}{l}\text { class group of Weil divisor } \\
\text { classes }\end{array}$ & Pic $X$ & Picard group of invertible modules \\
$\mathrm{B}(X)$ & $\begin{array}{l}\text { Brauer group of Azumaya } \\
\text { algebra classes }\end{array}$ & $\mathbb{G}_{m}$ & étale sheaf of units \\
$\rho_{0}$ & $=\operatorname{dim}_{\mathbb{Q}}\left[H^{0}\left(X_{\text {ét }}, \mathbb{G}_{m}\right) \otimes \mathbb{Q}\right]$ & $\rho_{i}$ & $=\operatorname{dim}_{\mathbb{Q}}\left[H^{i}\left(X_{\text {ét }}, \mathbb{G}_{m}\right) \otimes \mathbb{Q}\right]($ for $i>0)$ \\
$\rho_{1}^{\prime}$ & $=\operatorname{dim}_{\mathbb{Q}}[\mathrm{Cl}(X) \otimes \mathbb{Q}]$ & $\kappa_{i}$ & $=\operatorname{dim}_{\mathbb{Q}}\left[H^{i}\left(\Delta_{\text {top }}, \mathcal{S F}\right) \otimes \mathbb{Q}\right]($ for $i \geq 0)$ \\
$s$ & $=\operatorname{dim}_{\mathbb{R}} \mathbb{R}\left|\Delta_{\text {fan }}\right|$ & $\Delta(i)$ & $=\{\sigma \in \Delta \mid \operatorname{dim} \sigma=i\}$ \\
$K$ & $=K(X)$ the function field of $X$ & $\mathcal{S F}$ & sheaf of $\Delta$-linear support functions \\
$\mathcal{W}$ & sheaf of Weil divisors & $\mathcal{P}$ & quotient sheaf $\mathcal{W} / \mathcal{S F}$ \\
\hline
\end{tabular}

\section{FAN INVARIANTS}

In Theorem 2.3 we determine the Brauer group of a nonsingular toric variety over $k$. This invariant depends on $k$. We then show in Theorem 2.5 that $\mathrm{Cl}(X)$, Pic $X$ and the relative cohomology group $H^{2}\left(K / X_{\text {ét }}, \mathbb{G}_{m}\right)$ depend only on $\Delta_{\text {fan }}$, not on $k$. In order to determine the Brauer group of a nonsingular toric variety over $k$, we use the following theorem of Hoobler. 
Theorem 2.1. Let $R=A\left[x_{1}, x_{1}^{-1}, \ldots, x_{r}, x_{r}^{-1}\right]$, where $A$ is a connected, normal integral domain. Suppose $\nu$ is an integer relatively prime to the residue characteristics of $A$. Then

$$
H^{1}(R, \mathbb{Z} / \nu)=H^{1}(A, \mathbb{Z} / \nu) \oplus\left(\bigoplus^{r} \mu_{\nu}^{-1}\right)
$$

and

$$
{ }_{\nu} \mathrm{B}(R)={ }_{\nu} \mathrm{B}(A) \oplus\left(\bigoplus^{r} H^{1}(A, \mathbb{Z} / \nu)\right) \oplus\left(\bigoplus^{r(r-1) / 2} \mu_{\nu}^{-1}\right) .
$$

Proof. See [9, Cor. 2.6].

Therefore the $\nu$-torsion of the Brauer group of $R=A\left[x_{1}, x_{1}^{-1}, \ldots, x_{r}, x_{r}^{-1}\right]$ is generated by the Azumaya $A$-algebras and the classes of cyclic crossed product algebras of 2 types. For each cyclic Galois extension $C / A$ of degree $\nu$ with group $\langle\sigma\rangle$ and for each $1 \leq i \leq r$, there is the cyclic crossed product $\left(C / R,\langle\sigma\rangle, x_{i}\right)$ which is an Azumaya algebra over $R$. If there exists a primitive $\nu$-th root of unity $\zeta$ over $A$, then the symbol algebras $\left(x_{i}, x_{j}\right)_{\nu}$ are Azumaya algebras over $R$.

Example 2.2. Let $R=\mathbb{R}\left[x_{1}, x_{1}^{-1}, \ldots, x_{r}, x_{r}^{-1}\right]$. Then by Theorem $2.1 \mathrm{~B}(R)$ is an elementary 2-group and

$$
\begin{aligned}
\mathrm{B}(R) & \cong \mathrm{B}(\mathbb{R}) \oplus\left(\bigoplus^{r} H^{1}(\mathbb{R}, \mathbb{Z} / 2)\right) \oplus\left(\stackrel{r(r-1) / 2}{\bigoplus} \mu_{2}^{-1}\right) \\
& \cong(\mathbb{Z} / 2)^{1+r+r(r-1) / 2}
\end{aligned}
$$

Define a sheaf $\mathcal{S F}$ on $\Delta_{\text {top }}$ by assigning to each open set $\Delta^{\prime} \subseteq \Delta_{\text {top }}$ the abelian group $\operatorname{SF}\left(\Delta^{\prime}\right)$ of support functions on $\Delta^{\prime}$. Let $M=\operatorname{Hom}(N, \mathbb{Z})$ be the dual of $N$. There is a natural map $M \rightarrow \mathrm{SF}\left(\Delta^{\prime}\right)$ which is locally surjective. If $\mathcal{M}$ denotes the constant sheaf of $M$ on $\Delta_{t o p}$, then there is an exact sequence of sheaves on $\Delta_{t o p}$ :

$$
0 \rightarrow \mathcal{U} \rightarrow \mathcal{M} \rightarrow \mathcal{S F} \rightarrow 0
$$

where $\mathcal{U}$ is defined by the sequence (6). On any open $\Delta^{\prime} \subseteq \Delta_{\text {top }}, \mathcal{U}\left(\Delta^{\prime}\right)=\left|\Delta^{\prime}\right|^{\perp} \cap M=$ $\left\{m \in M \mid\langle m, y\rangle=0\right.$ for all $\left.y \in\left|\Delta^{\prime}\right|\right\}$. Because $\mathcal{M}$ is flasque, $H^{p}\left(\Delta_{\text {top }}, \mathcal{M}\right)=0$ for all $p \geq 1$, so $H^{p}\left(\Delta_{t o p}, \mathcal{S F}\right) \cong H^{p+1}\left(\Delta_{t o p}, \mathcal{U}\right)$ for all $p \geq 1$.

Let $k$ be a field and $X=T_{N} \operatorname{emb}(\Delta, k)$ a nonsingular toric variety over $k$. Let $N^{\prime}=$ $\left\langle\bigcup_{\sigma \in \Delta} \sigma \cap N\right\rangle$, let $\nu \geq 2$ be relatively prime to char $k$, and let $M_{\nu}=\left\{m \in M \mid\left\langle m, n^{\prime}\right\rangle \equiv 0\right.$ $(\bmod \nu)$ for all $\left.n^{\prime} \in N^{\prime}\right\}$. The basis theorem for finitely generated abelian groups gives a basis $n_{1}, \ldots, n_{r}$ of $N$ such that $N^{\prime}=\mathbb{Z} a_{1} n_{1} \oplus \mathbb{Z} a_{2} n_{2} \oplus \cdots \oplus \mathbb{Z} a_{r} n_{r}$ where the $a_{i}$ are nonnegative integers and $a_{i} \mid a_{i+1}$ for $1 \leq i \leq r-1$. As in [3] call $a_{1}, \ldots, a_{r}$ the set of invariant factors of $X$. 
Theorem 2.3. In the above terminology, if $\left(\nu, a_{i}\right)$ is the greatest common divisor of $\nu$ and $a_{i}$, then

$$
\begin{gathered}
H^{1}(X, \mathbb{Z} / \nu) \cong H^{1}(k, \mathbb{Z} / \nu) \oplus\left(M_{\nu} / \nu M \otimes \mu_{\nu}^{-1}\right) \\
\cong H^{1}(k, \mathbb{Z} / \nu) \oplus\left(\bigoplus_{i=1}^{r} \mathbb{Z} /\left(\nu, a_{i}\right) \otimes \mu_{\nu}^{-1}\right) . \\
{ }_{\nu} \mathrm{B}(X)={ }_{\nu} \mathrm{B}^{\prime}(X) \cong \\
{ }_{\nu} \mathrm{B}(k) \oplus\left(\bigoplus_{i=1}^{r} H^{1}(k, \mathbb{Z} / \nu) \otimes \mathbb{Z} /\left(\nu, a_{i}\right)\right) \oplus\left(\bigoplus_{i=1}^{r} \operatorname{Hom}\left(\mathbb{Z} / a_{i} \otimes \mu_{\nu}, \mathbb{Q} / \mathbb{Z}\right)^{r-i}\right)
\end{gathered}
$$

Proof. Follows from the proof of [3, Theorem 1.1] and Theorem 2.1.

Therefore the $\nu$-torsion of the Brauer group of the nonsingular toric variety $X$ is generated by the classes of algebras from $k$ and cyclic crossed product algebras of 2 types. For each cyclic Galois extension $C / k$ of degree $\nu$ with group $\langle\sigma\rangle$ and for each $1 \leq i \leq r$, there is the cyclic crossed product $\left(C / k,\langle\sigma\rangle, x_{i}\right)$ which is an Azumaya algebra over the torus $T_{N}$. This algebra is unramified on $X$ if and only if the function $x_{i}$ corresponds to an element of $M_{\nu}$. If there exists a $\nu$-th root of unity $\zeta$ over $k$, then the symbol algebras $\left(x_{i}, x_{j}\right)_{\nu}$ are Azumaya algebras over $T_{N}$. Those symbols which are unramified on $X$ correspond to the last summand of (8).

Example 2.4. Let $k=\mathbb{R}$ and $X=T_{N} \operatorname{emb}(\Delta)$ a nonsingular toric variety over $\mathbb{R}$. Then by Theorem 2.3 $\mathrm{B}(X)$ is an elementary 2-group. If $t=\left|\left\{a_{i} \mid\left(2, a_{i}\right) \neq 1\right\}\right|$, then

$$
\begin{aligned}
\mathrm{B}(X) & \cong \mathrm{B}(\mathbb{R}) \oplus\left(\bigoplus_{i=1}^{r} \mathbb{Z} /\left(2, a_{i}\right)\right) \oplus\left(\bigoplus_{i=1}^{r} \operatorname{Hom}\left(\mathbb{Z} / a_{i} \otimes \mu_{2}, \mathbb{Q} / \mathbb{Z}\right)^{r-i}\right) \\
& \cong \mathbb{Z} / 2 \oplus(\mathbb{Z} / 2)^{t} \oplus(\mathbb{Z} / 2)^{t(t-1) / 2}
\end{aligned}
$$

Theorem 2.5. Let $k$ be a field and $X=T_{N} \operatorname{emb}(\Delta)$ a toric variety over $k$ with function field $K$. Then

1. $H^{p}\left(\Delta_{\text {top }}, \mathcal{U}\right) \cong H^{p}\left(X_{\mathrm{Zar}}, \mathcal{O}^{*}\right)$ for all $p \geq 1$ hence $H^{p}\left(X_{\mathrm{Zar}}, \mathcal{O}^{*}\right)$ depends only on $\Delta_{\text {fan }}$, not $k$. In particular $\mathrm{Cl}(X)$ and $\mathrm{Pic} X$ depend only on $\Delta_{\text {fan }}$.

2. $H^{1}\left(\Delta_{\text {top }}, \mathcal{S F}\right) \cong H^{2}\left(X_{\mathrm{Zar}}, \mathcal{O}^{*}\right) \cong H^{2}\left(K / X_{\text {ét }}, \mathbb{G}_{m}\right)$ hence $H^{2}\left(K / X_{\text {ét }}, \mathbb{G}_{m}\right)$ depends only on $\Delta_{\text {fan }}$, not $k$.

3. If $\tilde{\Delta}$ is a nonsingular subdivision of $\Delta$ and $\tilde{X}=T_{N} \operatorname{emb}(\tilde{\Delta})$, then the sequence

$$
0 \rightarrow H^{2}\left(K / X_{\text {ét }}, \mathbb{G}_{m}\right) \rightarrow H^{2}\left(X_{\text {ét }}, \mathbb{G}_{m}\right) \rightarrow H^{2}\left(\tilde{X}_{\text {ét }}, \mathbb{G}_{m}\right) \rightarrow 0
$$

(with natural maps) is split-exact.

Proof. The theorem follows from [4], noting that the proof of [4, Theorem 1] did not assume that $k$ is algebraically closed until the proof of Lemma 7 where it was not necessary anyway. 


\section{TOpological Invariants}

The first invariants to be considered as candidates for topological invariants are the following. Let $\Delta$ and $X$ be as in the Introduction. For each $i \geq 0$ we define a positive integer $\rho_{i}$. Set

$$
\begin{gathered}
\rho_{0}=\operatorname{dim}_{\mathbb{Q}}\left[H^{0}\left(X_{\text {ét }}, \mathbb{G}_{m}\right) / k^{*} \otimes \mathbb{Q}\right] \\
\rho_{1}=\operatorname{dim}_{\mathbb{Q}}\left[H^{1}\left(X_{\text {ét }}, \mathbb{G}_{m}\right) \otimes \mathbb{Q}\right]=\operatorname{dim}_{\mathbb{Q}}(\operatorname{Pic} X \otimes \mathbb{Q})
\end{gathered}
$$

and for $i \geq 2$,

$$
\rho_{i}=\operatorname{dim}_{\mathbb{Q}}\left[H^{i}\left(X_{\text {ét }}, \mathbb{G}_{m}\right) \otimes \mathbb{Q}\right] .
$$

The number $\rho_{1}$ is the traditional Picard number $\rho$ associated to $X$. Also set

$$
\rho_{1}^{\prime}=\operatorname{dim}_{\mathbb{Q}}[\mathrm{Cl}(X) \otimes \mathbb{Q}] .
$$

It follows from Theorem 3.1 below that $\rho_{0}$ is a fan invariant and from Theorem 2.5 above that $\rho_{1}, \rho_{1}^{\prime}$, and $\rho_{2}$ are fan invariants. Since $\Delta$ is finite, $\rho_{0}, \rho_{1}, \rho_{1}^{\prime}$ and $\rho_{2}$ are finite. For $\rho_{0}$, $\rho_{1}$ and $\rho_{1}^{\prime}$ see [10] or [6]. For $\rho_{2}$ this follows from [4].

Examples where the number $\rho_{2}$ is computed seem to be somewhat scarce. Grothendieck [7, II] and Childs [1] each give an example of a local ring $\mathcal{O}_{x}$ on a normal surface where $H^{2}\left(\left(\mathcal{O}_{x}\right)_{\text {ét }}, \mathbb{G}_{m}\right)$ is torsion free, but in each case $H^{2}\left(\left(\mathcal{O}_{x}\right)_{\text {ét }}, \mathbb{G}_{m}\right)$ is not finitely generated.

Remark 1. The dimension of the topological space $\Delta_{\text {top }}$ is defined to be the length of a maximal chain of irreducible closed subsets. One can check that this is equal to $\max \{\operatorname{dim} \sigma \mid$ $\sigma \in \Delta\}$. Therefore $\operatorname{dim} \Delta_{\text {top }}$ is a topological invariant of $\Delta_{\text {fan }}$.

Remark 2. Define another sequence of invariants by

$$
\kappa_{i}=\operatorname{dim}_{\mathbb{Q}}\left[H^{i}\left(\Delta_{t o p}, \mathcal{S F}\right) \otimes \mathbb{Q}\right]
$$

for $i \geq 0$. It follows from Theorem 2.5 (2) that $\kappa_{1}=\rho_{2}$. Let $\sigma_{0}, \ldots, \sigma_{m}$ be the maximal cones in $\Delta$. From [4, Lemma 8] $\kappa_{i}$ can be computed from the Čech complex

$$
0 \rightarrow \underset{i}{\oplus} \mathcal{S} \mathcal{F}\left(\sigma_{i}\right) \stackrel{\delta^{0}}{\rightarrow} \underset{i<j}{\oplus} \mathcal{S} \mathcal{F}\left(\sigma_{i j}\right) \stackrel{\delta^{1}}{\rightarrow} \underset{i<j<k}{\oplus} \mathcal{S} \mathcal{F}\left(\sigma_{i j k}\right) \rightarrow \ldots
$$

For any cone $\tau \in \Delta$, $\operatorname{dim}_{\mathbb{Q}}(\mathcal{S F}(\Delta(\tau)) \otimes \mathbb{Q})=\operatorname{dim} \tau$. Therefore, if $C^{i}$ denotes the $i$-th group of Čech cochains in $(10)$ and $c_{i}=\operatorname{dim}_{\mathbb{Q}}\left(C^{i} \otimes \mathbb{Q}\right)$, then the integer

$$
c_{0}-c_{1}+c_{2}-\ldots
$$

is a topological invariant of $\Delta_{f a n}$. Note that there exists an integer $M$ such that $C^{j}=0$ for all $j>M$. If $\operatorname{dim}\left(\Delta_{\text {top }}\right)=t$, then $\kappa_{j}=0$ for all $j>t$. So the left hand side of

$$
\kappa_{0}-\kappa_{1}+\ldots(-1)^{t} \kappa_{t}=c_{0}-c_{1}+c_{2}-\ldots(-1)^{M} c_{M}
$$

is a topological invariant of $\Delta_{f a n}$.

Remark 3. Let $\Delta$ be a finite fan on $N \otimes \mathbb{R}$ where $N=\mathbb{Z}^{r}$. Setting $s=\operatorname{dim}_{\mathbb{R}} \mathbb{R}\left|\Delta_{\text {fan }}\right|$, we see that $s$ is not a topological invariant of $\Delta_{\text {fan }}$. Since $s \geq \operatorname{dim} \Delta_{t o p}$, if $\operatorname{dim} \Delta_{t o p}=r$, then $s=r$ so if $\Delta$ contains a cone $\sigma$ such that $\operatorname{dim} \sigma=r$, then $s=r$ and $s$ is a topological invariant of $\Delta_{\text {fan }}$. This condition is satisfied, for instance, if $\Delta$ is a complete fan on $N \otimes \mathbb{R}$. 
Remark 4. Let $\sigma \in \Delta$ and let $\Delta(\sigma)$ denote the subfan of $\Delta$ consisting of the cone $\sigma$ and all of its faces. Then

$$
\operatorname{dim} \sigma=\operatorname{dim} \Delta(\sigma)_{t o p}
$$

so the dimensions of the cones in $\Delta$ depend only on $\Delta_{\text {top }}$. In particular, the number of 1-dimensional cones in $\Delta_{f a n}$ is a topological invariant.

Remark 5. The fan $\Delta_{f a n}$ is complete by definition if $\left|\Delta_{f a n}\right|=\mathbb{R}^{r}$. This is true if and only if

(i) $\Delta(r) \neq \emptyset$ and

(ii) for each cone $\sigma \in \Delta(r)$ and every $r$-1-dimensional face $\tau$ of $\sigma$ there is a cone $\sigma_{1} \in \Delta(r)$ such that $\tau=\sigma \cap \sigma_{1}$.

But these two conditions depend only on $\Delta_{t o p}$. That is, completeness can be thought of as a topological property of $\Delta_{\text {fan }}$.

From the next theorem, which combines some results on $\rho_{0}$ and $\rho_{1}^{\prime}$, we see that $\rho_{0}$ depends only on the dimension of the subspace spanned by $\left|\Delta_{\text {fan }}\right|$.

Theorem 3.1. Let $N=\mathbb{Z}^{r}, \Delta$ a fan on $N \otimes \mathbb{R}, X=T_{N} \operatorname{emb}(\Delta)$, and $s=\operatorname{dim}_{\mathbb{R}} \mathbb{R}\left|\Delta_{\text {fan }}\right|$ (that is, $s$ is the dimension of the $\mathbb{R}$-vector space spanned by the vectors in the support $\left.\left|\Delta_{\text {fan }}\right|\right)$. Then

(a) $\rho_{0}=r-s$, hence is a fan invariant, but not a topological invariant.

(b) Suppose $\Delta_{\text {fan }}$ contains a cone $\sigma$ such that $\operatorname{dim} \sigma=r$. This is true for example if $\Delta_{\text {fan }}$ is a complete fan on $N \otimes \mathbb{R}$. Then $\rho_{0}=0$ and $\rho_{0}$ is a topological invariant of $\Delta_{\text {fan }}$.

(c) $\rho_{1}^{\prime}=\operatorname{dim}_{\mathbb{Q}}(\mathrm{Cl}(X) \otimes \mathbb{Q})=\#(\Delta(1))-s$. If $\operatorname{dim} \Delta_{\text {top }}=r$, then $\rho_{1}^{\prime}$ is a topological invariant of $\Delta_{\text {fan }}$.

(d) The number $\rho_{0}-\rho_{1}^{\prime}$ is a topological invariant of $\Delta_{\text {fan }}$.

Proof. (a) Let $N_{1}=N \cap \mathbb{R}\left|\Delta_{\text {fan }}\right|, M_{1}=\operatorname{Hom}_{\mathbb{Z}}\left(N_{1}, \mathbb{Z}\right), M_{2}=N_{1}^{\perp}, N_{2}=\operatorname{Hom}_{\mathbb{Z}}\left(M_{2}, \mathbb{Z}\right)$. Then $M=M_{1} \oplus M_{2}$ and $N=N_{1} \oplus N_{2}$. Viewing $\Delta$ as a fan on the $s$-dimensional vector space $N_{1} \otimes \mathbb{R}, X=T_{N_{1}} \operatorname{emb}(\Delta) \times T_{N_{2}}$ and $H^{0}\left(X, \mathbb{G}_{m}\right) / k^{*} \cong H^{0}\left(T_{N_{2}}, \mathbb{G}_{m}\right) / k^{*} \cong \mathbb{Z}^{r-s}$. So $\rho_{0}=r-s$.

(b) In this case $s=\operatorname{dim}_{\mathbb{R}} \mathbb{R}\left|\Delta_{\text {fan }}\right|=\operatorname{dim}_{\mathbb{R}}(\mathbb{R} \sigma)=\operatorname{dim} \sigma=r$.

(c) Let $N_{0}=N \cap \mathbb{R}\left|\Delta_{\text {fan }}\right|$ be the set of lattice points in the subspace $\mathbb{R}\left|\Delta_{\text {fan }}\right|$ and $M_{0}=$ $\operatorname{Hom}_{\mathbb{Z}}\left(N_{0}, \mathbb{Z}\right)$. Then $\operatorname{dim}_{\mathbb{Q}}\left(M_{0} \otimes \mathbb{Q}\right)=\operatorname{dim}_{\mathbb{Q}}\left(N_{0} \otimes \mathbb{Q}\right)=\operatorname{dim}_{\mathbb{R}} \mathbb{R}\left|\Delta_{\text {fan }}\right|=s$. From [10, Corollary 2.5] there is a presentation of $\mathrm{Cl}(X)$

$$
0 \rightarrow M_{0} \rightarrow \bigoplus_{\rho \in \Delta(1)} \mathbb{Z} \rho \rightarrow \mathrm{Cl}(X) \rightarrow 0
$$

So $\operatorname{dim}_{\mathbb{Q}}(\mathrm{Cl}(X) \otimes \mathbb{Q})=\#(\Delta(1))-s$. In particular, if $\operatorname{dim} \Delta_{\text {top }}=r$, then $r=s$ so $\operatorname{dim}_{\mathbb{Q}}(\mathrm{Cl}(X) \otimes \mathbb{Q})$ is a topological invariant.

(d) From (a) and (c),

$$
\rho_{0}-\rho_{1}^{\prime}=(r-s)-(\#(\Delta(1))-s)=r-\#(\Delta(1))
$$

which depends only on $\Delta_{t o p}$.

Remark 6. Let $N=\mathbb{Z}^{r}, \Delta$ a fan on $N \otimes \mathbb{R}, s=\operatorname{dim}_{\mathbb{R}} \mathbb{R}\left|\Delta_{\text {fan }}\right|, t=\max _{\sigma \in \Delta}\{\operatorname{dim} \sigma\}$. It follows from [3, Theorem 2.3] that if $t \leq 2$, then $\rho_{1}=\#(\Delta(1))-s$ and $\rho_{2}=0$. In this case $\Delta$ is a simplicial fan, so this is a special case of the following theorem. 
Theorem 3.2. Let $N=\mathbb{Z}^{r}$. Let $\Delta$ be a simplicial fan on $N \otimes \mathbb{R}$ and $s=\operatorname{dim}_{\mathbb{R}} \mathbb{R}\left|\Delta_{\text {fan }}\right|$. Then

(a) $\rho_{1}=\#(\Delta(1))-s$.

(b) $\rho_{2}=0$ hence is a topological invariant of $\Delta_{\text {fan }}$.

(c) If $\operatorname{dim} \Delta_{\text {top }}=r$, then $\rho_{1}$ is a topological invariant of $\Delta_{\text {fan }}$.

(d) $\rho_{0}-\rho_{1}+\rho_{2}$ is a topological invariant of $\Delta_{\text {fan }}$.

Proof. Suppose $\sigma \in \Delta$ is a simplicial cone and $\operatorname{dim} \sigma=d$. For any support function $h \in$ $\mathcal{S F}(\Delta),\left.h\right|_{\sigma}$ is linear and completely determined by its values on a spanning set $\left\{\eta_{1}, \ldots, \eta_{d}\right\} \subseteq$ $N$ for $\sigma$. Since $\operatorname{dim} \sigma=d, \sigma$ is spanned by $d$ lattice points. So $\mathcal{S F}(\Delta(\sigma)) \otimes \mathbb{Q} \cong \mathbb{Q}^{d}$.

If $\tau_{0}, \ldots, \tau_{n}$ are the cones in $\Delta(1)$, and $\Gamma=\left\{0, \tau_{0}, \ldots, \tau_{n}\right\}$, then $\Gamma_{\text {top }}$ is an open subset of the topological space $\Delta_{\text {top }}$. Define the sheaf $\mathcal{W}$ on $\Delta_{\text {top }}$ to be the direct image $i_{*}\left(\left.\mathcal{S F}\right|_{\Gamma_{\text {top }}}\right)$. Since $\Gamma_{\text {fan }}$ is a nonsingular fan, $\left.\mathcal{S} \mathcal{F}\right|_{\Gamma_{\text {top }}}$ is the sheaf defined by $\Xi \mapsto \mathbb{Z}^{\#(\Xi(1))}$ for each open $\Xi \subseteq \Delta_{\text {top }}$. It follows that $\mathcal{W}(\Xi)=\mathbb{Z}^{\#(\Xi(1))}$, hence $\mathcal{W}$ is a flasque sheaf. So there is an embedding $\mathcal{S F} \rightarrow \mathcal{W}$ of sheaves on $\Delta_{\text {top }}$ and we define $\mathcal{P}$ by the exact sequence of sheaves $\llbracket$, (13), p. 149]

$$
0 \rightarrow \mathcal{S F} \rightarrow \mathcal{W} \rightarrow \mathcal{P} \rightarrow 0
$$

Since $\Delta$ is simplicial, $\mathcal{S} \mathcal{F}(\Delta(\sigma))$ and $\mathcal{W}(\Delta(\sigma))$ are free of the same rank $\operatorname{dim} \sigma$. Therefore, $\mathcal{P}$ is locally torsion, hence torsion. Because $\mathcal{W}$ is flasque, $H^{1}\left(\Delta_{\text {top }}, \mathcal{W}\right)=0$ and the long exact sequence associated to (14) becomes

$$
0 \rightarrow H^{0}\left(\Delta_{\text {top }}, \mathcal{S F}\right) \rightarrow H^{0}\left(\Delta_{\text {top }}, \mathcal{W}\right) \rightarrow H^{0}\left(\Delta_{\text {top }}, \mathcal{P}\right) \rightarrow H^{1}\left(\Delta_{\text {top }}, \mathcal{S} \mathcal{F}\right) \rightarrow 0 .
$$

Because $\mathcal{P}$ is torsion, $H^{0}\left(\Delta_{\text {top }}, \mathcal{P}\right)$ is torsion. So $H^{1}\left(\Delta_{\text {top }}, \mathcal{S F}\right) \otimes \mathbb{Q}=0$. By [4, Theorem 1] $\rho_{2}=\operatorname{dim}\left(H^{1}\left(\Delta_{\text {top }}, \mathcal{S F}\right) \otimes \mathbb{Q}\right)=0$. This proves (b). It also follows from (15) that we obtain the isomorphism of [10, Proposition 2.1(v), p. 69]

$$
H^{0}\left(\Delta_{\text {top }}, \mathcal{S F}\right) \otimes \mathbb{Q} \cong H^{0}\left(\Delta_{\text {top }}, \mathcal{W}\right) \otimes \mathbb{Q} \text {. }
$$

By [⿴囗十, Lemma 8] there is an exact sequence

$$
0 \rightarrow M_{0} \rightarrow \mathcal{S F}\left(\Delta_{t o p}\right) \rightarrow \operatorname{Pic} X \rightarrow 0 .
$$

Combining (13) and (17), we have a commutative diagram with exact rows and columns.

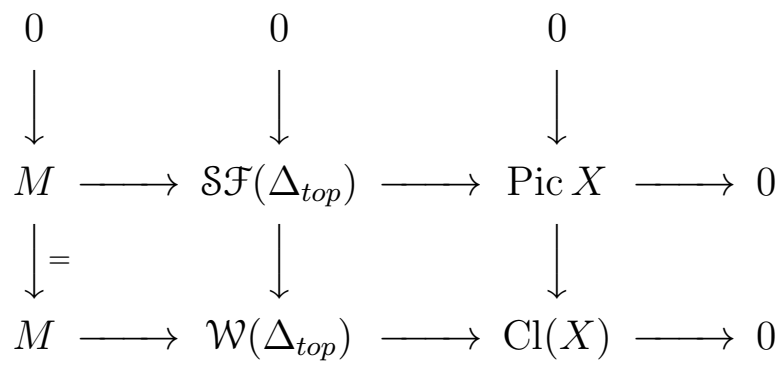

Because the center vertical arrow in (18) tensored with $\mathbb{Q}$ is the isomorphism (16), from (18) it follows that

$$
\operatorname{Pic}(X) \otimes \mathbb{Q} \cong \operatorname{Cl}(X) \otimes \mathbb{Q} .
$$

It follows from Theorem 3.1 that $\rho_{1}=\#(\Delta(1))-s$. This proves (a). In case $(\mathrm{c}), s=r$ so $\rho_{1}$ is a topological invariant. 
(d) From Theorem 3.1 and parts (a) and (b),

$$
\rho_{0}-\rho_{1}+\rho_{2}=(r-s)-(\#(\Delta(1))-s)+0=r-\#(\Delta(1))
$$

which only depends on $\Delta_{t o p}$.

Lemma 3.3. For any cone $\sigma \in \Delta$, let $\Delta(\sigma)$ denote the subfan of $\Delta$ consisting of $\sigma$ and all of its faces and $U_{\sigma}=T_{N} \operatorname{emb}(\Delta(\sigma))$. Then $H^{0}\left(\Delta(\sigma)_{\text {top }}, \mathcal{P}\right)=\mathrm{Cl}\left(U_{\sigma}\right)$.

Proof. For each $\sigma \in \Delta$ we have $H^{1}\left(\Delta(\sigma)_{t o p}, \mathcal{S F}\right)=0$ [4, Lemma 2.a, p. 139] so from (15)

$$
H^{0}\left(\Delta(\sigma)_{t o p}, \mathcal{P}\right)=\mathcal{W}\left(\Delta(\sigma)_{t o p}\right) / \mathcal{S F}\left(\Delta(\sigma)_{t o p}\right) .
$$

Now $\mathcal{W}\left(\Delta(\sigma)_{\text {top }}\right)=\mathbb{Z}^{\#(\Delta(\sigma)(1))}$ and support functions are linear on a cone $\sigma$, so

$$
\mathcal{W}\left(\Delta(\sigma)_{\text {top }}\right) / \mathcal{S F}\left(\Delta(\sigma)_{\text {top }}\right) \cong \mathbb{Z}^{\#(\Delta(\sigma)(1))} / \operatorname{im}(M)=\mathrm{Cl}\left(U_{\sigma}\right) .
$$

Lemma 3.4. Let $\sigma$ be a cone in $N \otimes \mathbb{R}$ and $s=\operatorname{dim} \sigma . \quad$ Then $\operatorname{dim}_{\mathbb{Q}}\left(\mathrm{Cl}\left(U_{\sigma}\right) \otimes \mathbb{Q}\right)=$ $\#(\Delta(\sigma)(1))-s$. Also $\sigma$ is simplicial if and only if $\mathrm{Cl}\left(U_{\sigma}\right)$ is torsion.

Proof. Follows from Theorem 3.1.

The following can be considered a theorem for 3-dimensional fans.

Theorem 3.5. Let $\Delta$ be a fan on $N \otimes \mathbb{R}$. Let $\sigma_{0}, \ldots, \sigma_{w}$ be the maximal cones in $\Delta$. Assume $\sigma_{i} \cap \sigma_{j}$ is simplicial for each $i \neq j$. These assumptions are satisfied for example if $\operatorname{dim} \Delta_{t o p} \leq 3$. Then

(a)

$$
\rho_{1}+s+\sum_{i=0}^{w}\left(\#\left(\Delta\left(\sigma_{i}\right)(1)\right)-s_{i}\right)=\rho_{2}+\#(\Delta(1))
$$

where we set $s_{i}=\operatorname{dim} \sigma_{i}$ for each $i=0, \ldots, w$ and $s=\operatorname{dim}_{\mathbb{R}} \mathbb{R}\left|\Delta_{\text {fan }}\right|$.

(b) $\rho_{0}-\rho_{1}+\rho_{2}$ is a topological invariant of $\Delta_{\text {fan }}$.

Proof. (a) The set $\left\{\Delta\left(\sigma_{i}\right)_{t o p}\right\}_{i=0}^{w}$ is an open cover of $\Delta_{\text {top }}$ and the sequence

$$
0 \rightarrow H^{0}\left(\Delta_{\text {top }}, \mathcal{P}\right) \rightarrow \bigoplus_{i=0}^{w} H^{0}\left(\Delta\left(\sigma_{i}\right)_{t o p}, \mathcal{P}\right) \rightarrow \bigoplus_{i=1}^{w} \bigoplus_{j=0}^{i-1} H^{0}\left(\Delta\left(\sigma_{i} \cap \sigma_{j}\right)_{t o p}, \mathcal{P}\right)
$$

is exact since $\mathcal{P}$ is a sheaf. Applying Lemma 3.3, the sequence (20) can be written

$$
0 \rightarrow H^{0}\left(\Delta_{\text {top }}, \mathcal{P}\right) \rightarrow \bigoplus_{i=0}^{w} \mathrm{Cl}\left(U_{\sigma_{i}}\right) \rightarrow \bigoplus_{i=1}^{w} \bigoplus_{j=0}^{i-1} \mathrm{Cl}\left(U_{\sigma_{i} \cap \sigma_{j}}\right)
$$

By our assumption $\sigma_{i} \cap \sigma_{j}$ is simplicial. By Lemma 3.4, $\mathrm{Cl}\left(U_{\sigma_{i} \cap \sigma_{j}}\right)$ is torsion. By «, Theorem 1] $H^{1}\left(\Delta_{t o p}, \mathcal{S F}\right) \cong H^{2}\left(K / X_{\text {ét }}, \mathbb{G}_{m}\right)$ and the torsion-free part of $H^{2}\left(X_{\text {ét }}, \mathbb{G}_{m}\right)$ is equal to the torsion-free part of $H^{2}\left(K / X_{\text {ét }}, \mathbb{G}_{m}\right)$. We compute the rank of the torsion-free part of $H^{2}\left(K / X_{\text {ét }}, \mathbb{G}_{m}\right)$ from (15) tensored with $\mathbb{Q}$ :

$$
0 \rightarrow \mathcal{S F}\left(\Delta_{\text {top }}\right) \otimes \mathbb{Q} \rightarrow \mathcal{W}\left(\Delta_{\text {top }}\right) \otimes \mathbb{Q} \rightarrow \mathcal{P}\left(\Delta_{\text {top }}\right) \otimes \mathbb{Q} \rightarrow H^{2}\left(K / X_{\text {ét }}, \mathbb{G}_{m}\right) \otimes \mathbb{Q} \rightarrow 0 .
$$




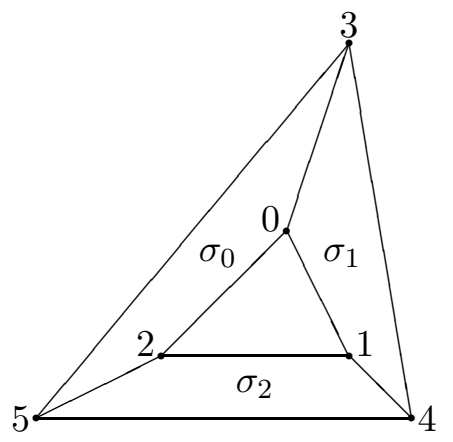

FiguRE 1.

Tensoring (17) with $\mathbb{Q}$ and counting dimensions we find $\operatorname{dim}_{\mathbb{Q}}\left(\mathcal{S F}\left(\Delta_{\text {top }}\right) \otimes \mathbb{Q}\right)=\operatorname{dim}_{\mathbb{Q}}(\operatorname{Pic} X$ $\otimes \mathbb{Q})+s=\rho_{1}+s$. By definition $\mathcal{W}\left(\Delta_{\text {top }}\right) \otimes \mathbb{Q}=\mathbb{Q}^{\#(\Delta(1))}$. From (21) $\mathcal{P}\left(\Delta_{\text {top }}\right) \otimes \mathbb{Q}=$ $\bigoplus_{i=0}^{w} \mathrm{Cl}\left(U_{\sigma_{i}}\right) \otimes \mathbb{Q}$. From Lemma 3.4, $\operatorname{dim}_{\mathbb{Q}}\left(\mathrm{Cl}\left(U_{\sigma_{i}}\right) \otimes \mathbb{Q}\right)=\#\left(\Delta\left(\sigma_{i}\right)(1)\right)-s_{i}$. Counting dimensions in (22), we have the equation

$$
\rho_{2}=\rho_{1}+s+\sum_{i=0}^{w}\left(\#\left(\Delta\left(\sigma_{i}\right)(1)\right)-s_{i}\right)-\#(\Delta(1)) .
$$

(b) From (a) and Theorem 3.1 we have

$$
\begin{aligned}
\rho_{0}-\rho_{1}+\rho_{2} & =(r-s)+s+\sum_{i=0}^{w}\left(\#\left(\Delta\left(\sigma_{i}\right)(1)\right)-s_{i}\right)-\#(\Delta(1)) \\
& =r+\sum_{i=0}^{w}\left(\#\left(\Delta\left(\sigma_{i}\right)(1)\right)-s_{i}\right)-\#(\Delta(1))
\end{aligned}
$$

which depends only on $\Delta_{t o p}$.

As the next example shows, $\rho_{1}$ and $\rho_{2}$ are not topological invariants of $\Delta_{\text {fan }}$ when $r \geq 3$ and $\Delta$ is not simplicial.

Example 3.6. Let $\Delta$ be a fan on $\mathbb{R}^{3}$ and suppose $\Delta$ consists of three cones of dimension 3 and 6 cones of dimension 1 such that for each $\sigma_{i} \in \Delta(3), \#\left(\Delta\left(\sigma_{i}\right)(1)\right)=4$. Assume that the intersection of the fan $\Delta$ with the unit sphere traces a graph that looks like that shown in Figure 11.

For any such fan $\Delta, \Delta_{\text {top }}$ is unique up to homeomorphism. We consider 2 such fans $\Delta$ and $\Delta^{\prime}$ such that $\rho_{1}(\Delta) \neq \rho_{1}\left(\Delta^{\prime}\right)$ and $\rho_{2}(\Delta) \neq \rho_{2}\left(\Delta^{\prime}\right)$.

For $\Delta$, take $\Delta(1)$ to be $\left\{\mathbb{R}_{\geq} \eta_{i} \mid i=0 . .5\right\}$ where $\left\{\eta_{0}, \ldots, \eta_{5}\right\}=$

$$
\left\{\left(\begin{array}{c}
1 \\
0 \\
-2
\end{array}\right),\left(\begin{array}{c}
-1 \\
2 \\
-2
\end{array}\right),\left(\begin{array}{l}
-1 \\
-2 \\
-2
\end{array}\right),\left(\begin{array}{l}
1 \\
0 \\
2
\end{array}\right),\left(\begin{array}{c}
-1 \\
2 \\
2
\end{array}\right),\left(\begin{array}{c}
-1 \\
-2 \\
2
\end{array}\right)\right\} .
$$

Using the methods of [5, Section 4] we find that $\rho_{1}(\Delta)=1$ and $\rho_{2}(\Delta)=1$.

For $\Delta^{\prime}$, take $\Delta^{\prime}(1)$ to be $\left\{\mathbb{R}_{\geq} \eta_{i} \mid i=0 . .5\right\}$ where $\left\{\eta_{0}, \ldots, \eta_{5}\right\}=$

$$
\left\{\left(\begin{array}{l}
0 \\
1 \\
1
\end{array}\right),\left(\begin{array}{l}
0 \\
0 \\
1
\end{array}\right),\left(\begin{array}{l}
1 \\
0 \\
1
\end{array}\right),\left(\begin{array}{c}
-1 \\
3 \\
1
\end{array}\right),\left(\begin{array}{c}
-2 \\
-1 \\
1
\end{array}\right),\left(\begin{array}{c}
3 \\
-1 \\
1
\end{array}\right)\right\} \text {. }
$$


Using the methods of [5, Section 4] we find that $\rho_{1}\left(\Delta^{\prime}\right)=0$ and $\rho_{2}\left(\Delta^{\prime}\right)=0$.

\section{A Stratification of the FiBERs of $\mathfrak{T}$}

Let $\Delta$ be a fan on $N \otimes \mathbb{R}=\mathbb{R}^{r}$ with $\Delta(1)=\left\{r_{0}, \ldots, r_{n}\right\}$. The intersection of $\Delta(1)$ with the unit sphere $S$ in $\mathbb{R}^{r}$ is a finite set of points, say $\left\{p_{0}, \ldots, p_{n}\right\}$. About each $p_{i}$ we can find an open ball $B_{i}$ on $S$ such that if $p_{i}$ is parametrized by $B_{i}$, then each choice of $\vec{p}=\left(p_{0}, p_{1}, \ldots, p_{n}\right)$ in $B_{0} \times B_{1} \times \cdots \times B_{n}$ defines a fan $\Phi=\Phi(\vec{p})$ such that $\Phi_{\text {top }} \cong \Delta_{\text {top }}$. The

manifold $B=\prod_{i=0}^{n} B_{i}$ parametrizes a subset of fans in the fiber $\mathfrak{T}^{-1}\left(\Delta_{\text {top }}\right)$. Call $B$ an open neighborhood of $\Delta$. If $\vec{p} \in B$, then the fan $\Phi=\Phi(\vec{p})$ is not necessarily rational. Sometimes it will be necessary to refer to points in $B$ that give rise to rational fans. In this case let

$$
\begin{aligned}
B_{\text {rat }}=\left\{\left(p_{0}, \ldots, p_{n}\right) \mid\right. & \text { for each } i, \\
& \left.p_{i} \text { is the intersection of a rational 1-dimensional cone } r_{i} \text { with } B_{i}\right\} .
\end{aligned}
$$

For the present section only we define the set of support functions on a fan to be a real vector space. If $\sigma$ is a cone, define $\mathcal{S F}(\sigma)$ to be $\operatorname{Hom}_{\mathbb{R}}(\mathbb{R} \sigma, \mathbb{R})$. Define $\mathcal{S} \mathcal{F}(\Delta)$ to be the kernel of $\delta^{0}$ in the Čech complex

$$
0 \rightarrow \underset{i}{\oplus} \mathcal{S} \mathcal{F}\left(\sigma_{i}\right) \stackrel{\delta^{0}}{\rightarrow} \underset{i<j}{\oplus} \mathcal{S} \mathcal{F}\left(\sigma_{i j}\right) \stackrel{\delta^{1}}{\rightarrow} \underset{i<j<k}{\oplus} \mathcal{S} \mathcal{F}\left(\sigma_{i j k}\right) \rightarrow \ldots
$$

where $\left\{\sigma_{0}, \ldots, \sigma_{w}\right\}$ is the set of maximal cones of $\Delta$. Define $\kappa_{0}(\Delta)=\operatorname{dim}_{\mathbb{R}} \mathcal{S} \mathcal{F}(\Delta)$. If $\Delta$ is a rational fan, then this definition of $\kappa_{0}$ agrees with the definition given in Remark 2 of Section 3. In this section we consider the stratification of the manifold $B$ by the invariant $\kappa_{0}$.

Example 4.1. If $\Delta$ is simplicial, then $\kappa_{0}=\#(\Delta(1))=n+1$ so $B$ has only 1 stratum. As was suggested in Example 3.6, we expect the stratification to be more interesting when $\Delta$ is nonsimplicial.

Example 4.2. Let $\Delta$ be the fan on $\mathbb{R}^{3}$ given in equation (24) of Example 3.6. Let $B=\prod_{i=0}^{5} B_{i}$ be an open neighborhood of $\Delta$. One can check that any support function $h \in \mathcal{S} \mathcal{F}(\Delta)$ is completely determined by its values on $r_{0}, r_{1}, r_{2}, r_{3}$ so $\kappa_{0}(\Delta) \leq 4$. From Example 3.6 we know that $\kappa_{0}(\Delta)=4$. It is possible to vary any one of the $r_{i}$ to achieve a fan $\Phi$ in $B$ with $\kappa=3$. So $B$ has exactly 2 strata. We will see later that the stratum where $\kappa_{0}=4$ is a Zariski closed subset of $B$.

Conjecture 4.3. Let $\Delta$ be a complete fan on $\mathbb{R}^{3}$ such that for each cone $\sigma \in \Delta(3)$, $\sigma$ is nonsimplicial. Let $B$ be an open neighborhood of $\Delta$ as described above. Then for a general choice of $\vec{p} \in B$, if $\Phi=\Phi(\vec{p})$, then every $\Phi$-linear support function is linear. In particular for a general choice of $\vec{p} \in B_{\text {rat }}, \kappa_{0}(\Phi)=3$ hence $\rho_{1}(\Phi)=0$ and $\rho_{2}(\Phi)$ is a topological invariant.

In Conjecture 4.3 by "general choice" of $\vec{p}$ we mean that there is a dense open subset $G \subseteq B$ and each fan in the set $\{\Phi(\vec{p}) \mid \vec{p} \in G\}$ satisfies the conjecture. That is, if Conjecture 4.3 is true, a sufficiently general fan $\Delta^{\prime}$ with $\Delta_{\text {top }}^{\prime} \cong \Delta_{\text {top }}$ should satisfy $\kappa_{0}\left(\Delta^{\prime}\right)=3$. 
As motivation for Conjecture 4.3, consider the case where each $\sigma \in \Delta(3)$ has exactly 4 1-dimensional faces. Let $\Delta(3)=\left\{\sigma_{0}, \ldots, \sigma_{w}\right\}, \Delta(2)=\left\{\tau_{0}, \ldots, \tau_{e}\right\}, \Delta(1)=\left\{r_{0}, \ldots, r_{n}\right\}$. The intersections of the cones in $\Delta(2)$ with the unit sphere $S$ in $\mathbb{R}^{3}$ trace out the edges of a graph on $S$. This graph has $e+1$ edges, $n+1$ vertices and $w+1$ regions. So $w+1=(e+1)-(n+1)+2$. Each $\sigma_{j}$ has exactly $4 \tau_{i}$ 's and each $\tau_{i}$ is in exactly $2 \sigma_{j}$ 's, so $2(e+1)=4(w+1)$ or $e+1=2(w+1)$. Hence $w+1=n-1$. From Theorem $3.1(\mathrm{c}) \rho_{1}^{\prime}=\operatorname{dim}_{\mathbb{Q}}(\mathrm{Cl}(X) \otimes \mathbb{Q})=$ $(n+1)-3=n-2$ and $\rho_{1}^{\prime}\left(\Delta\left(\sigma_{i}\right)\right)=\operatorname{dim}_{\mathbb{Q}}\left(\mathrm{Cl}\left(U_{\sigma_{i}}\right) \otimes \mathbb{Q}\right)=4-3=1$. From (22) we have an exact sequence

$$
0 \rightarrow \mathcal{S} \mathcal{F}\left(\Delta_{\text {top }}\right) \otimes \mathbb{Q} \rightarrow \mathcal{W}\left(\Delta_{\text {top }}\right) \otimes \mathbb{Q} \rightarrow \bigoplus_{i=0}^{w}\left(\mathrm{Cl}\left(U_{\sigma_{i}}\right) \otimes \mathbb{Q}\right)
$$

Since linear Weil divisors correspond to linear Cartier divisors (27) gives rise to

$$
0 \rightarrow \operatorname{Pic}(X) \otimes \mathbb{Q} \rightarrow \mathrm{Cl}(X) \otimes \mathbb{Q} \rightarrow \bigoplus_{i=0}^{w}\left(\mathrm{Cl}\left(U_{\sigma_{i}}\right) \otimes \mathbb{Q}\right)
$$

In (28) the middle term has dimension $n-2$ and the third term dimension $n-1$. For each $i$ the map $\mathrm{Cl}(X) \rightarrow \mathrm{Cl}\left(U_{\sigma_{i}}\right)$ is surjective. So we can view $\operatorname{Pic}(X) \otimes \mathbb{Q}$ as the intersection of $n-1$ hyperplanes through $(0)$ in $\mathbb{Q}^{n-2}$. In general, this intersection should be $(0)$. If the conclusion of Conjecture 4.3 is satisfied, then from (23) it follows that $\rho_{2}=3+(w+1)-(n+1)$ (for a general choice of $\Delta_{f a n}$ ).

Next we give an algorithm for computing an upper bound for $\kappa_{0}$ for a fan of arbitrary dimension. The algorithm can also be used to obtain an upper bound for $\rho_{1}$ and $\rho_{2}$ for complete rational 3 -dimensional fans. If $\Delta$ is a fan on $\mathbb{R}^{3}$ which contains at least 1 cone of dimension 3 , then $\rho_{0}=0$ and by Theorem 3.5 we have $\rho_{1}=\rho_{2}+$ (topological invariant). In this setting $\rho_{1}=\kappa_{0}-3$.

Algorithm 4.4. Let $\Delta$ be a fan on $N \otimes \mathbb{R}$. The following is an algorithm for computing an upper bound for $\kappa_{0}$.

The algorithm is based on the fact that the map $\mathcal{S F}(\Delta) \rightarrow \mathbb{Z}^{\#(\Delta(1))}$ is injective. The algorithm finds a subset $G$ of $\Delta(1)$ such that any support function $h$ in $\mathcal{S F}(\Delta) \otimes \mathbb{Q}$ is completely determined by its values on the 1-dimensional cones in $G$.

If $\sigma$ is a maximal cone in $\Delta$ of dimension $d$, then a support function $h$ is determined by its values on any $d$-dimensional faces of $\sigma$ that span a $d$-dimensional subspace of $N \otimes \mathbb{R}$. Pick $d$ such elements of $\sigma(1)$ and place them in a set called $G$. Place all other elements of $\sigma(1)$ in a set called $R$. Initially, $G$ and $R$ are both empty, and the starting cone $\sigma$ is chosen somewhat arbitrarily. The algorithm proceeds to branch from the starting cone $\sigma$ outward until all maximal cones of $\Delta$ have been visited and $\Delta(1)$ has been partitioned into $\Delta(1)=G \cup R$. The order in which the maximal cones are traversed is somewhat arbitrary and may affect both the resulting set $G$ and the resulting cardinality of $G$.

Step 0. Set $B=\{\sigma \in \Delta \mid \sigma$ is a maximal cone in $\Delta\}$. Set $G=\emptyset$ and $R=\emptyset$. Go to Step 3.

Step 1. If there is a maximal cone $\sigma \in B$ such that $\sigma(1) \cap(G \cup R)$ contains a spanning set for $\mathbb{R} \sigma$, then add the remaining cones in $\sigma(1)-G-R$ to $R$. Remove $\sigma$ from $B$. repeat Step 1 until the condition is false.

Step 2. If there is a maximal cone $\sigma \in B$ such that $\sigma(1) \cap(G \cup R) \neq \emptyset$, then pick $\sigma \in B$ such that 
(a)

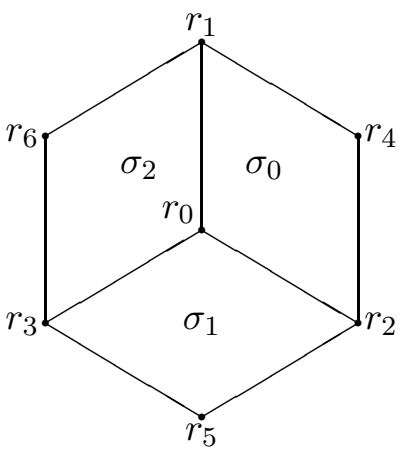

(b)

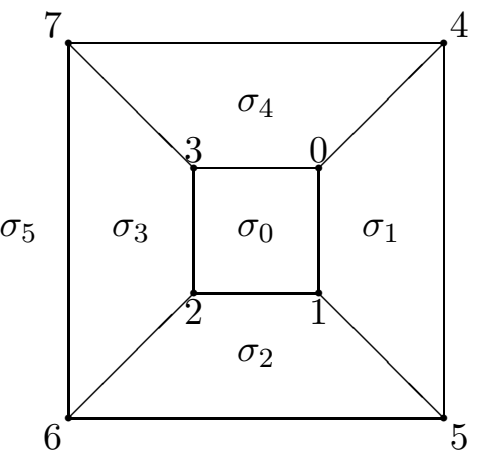

FiguRE 2.

(i) $e=\operatorname{dim}_{\mathbb{R}}\langle\sigma(1) \cap(G \cup R)\rangle$ is maximal and

(ii) $d=\operatorname{dim} \sigma$ is maximal among all $\sigma \in B$ satisfying (i).

For any $\sigma$ satisfying (i) and (ii), pick $\tau_{1}, \ldots, \tau_{e}$ in $\sigma(1) \cap(G \cup R)$ such that $\tau_{1}+\cdots+\tau_{e}$ has dimension $e$. Choose $\tau_{e+1}, \ldots, \tau_{d}$ in $\sigma(1)$ such that $\tau_{1}+\cdots+\tau_{d}$ has dimension $d$. Add $\tau_{e+1}, \ldots, \tau_{d}$ to $G$ and add the remaining elements $\sigma(1)-G-R-\left\{\tau_{e+1}, \ldots, \tau_{d}\right\}$ to $R$. Delete $\sigma$ from $B$. Go to Step 1 .

Step 3. If $B \neq \emptyset$, then pick $\sigma \in B$ such that $d=\operatorname{dim} \sigma$ is maximal. Pick $\tau_{1}, \ldots, \tau_{d}$ in $\sigma(1)$ such that $\tau_{1}+\cdots+\tau_{d}$ has dimension $d$. Add $\tau_{1}, \ldots, \tau_{d}$ to $G$ and add the remaining cones in $\sigma(1)-\left\{\tau_{1}, \ldots, \tau_{d}\right\}$ to $R$. Delete $\sigma$ from $B$. Go to Step 1 .

Step 4. This point is reached only if $B=\emptyset$. Now $\Delta(1)$ is partitioned into 2 sets: $\Delta(1)=G \cup R$. Any support function $h$ in $\mathcal{S F}(\Delta) \otimes \mathbb{Q}$ is determined completely by its values on $G$. So $\mathcal{S F}(\Delta) \rightarrow \mathbb{Z}^{\#(G)}$ is injective. Therefore $\#(G)$ is an upper bound for $\kappa_{0}$.

Example 4.5. Let $\Delta$ be a fan on $\mathbb{R}^{3}$ that consists of three 3-dimensional cones and assume that the intersection of the fan $\Delta$ with the unit sphere $S$ traces a graph as shown in Figure 2(a). In this example, we step through Algorithm 4.4 to see that $\kappa_{0}(\Delta) \leq 4$. It is shown later in Example 4.6 that for this fan, $\kappa_{0}=4$. Initially, $B=\left\{\sigma_{0}, \sigma_{1}, \sigma_{2}\right\}$ and $G=R=\emptyset$. The algorithm proceeds to Step 3. Place $r_{1}, r_{4}, r_{2}$ from $\sigma_{0}(1)$ in $G$ and $r_{0}$ in $R$. Delete $\sigma_{0}$ from $B$. The condition in Step 1 is still false, so the algorithm goes to Step 2. For $\sigma_{1}, r_{0}$ and $r_{2}$ are both in $G \cup R$ and $r_{0}+r_{2}$ has dimension $e=2$. Place $r_{5}$ in $G$ and $r_{3}$ in $R$. Delete $\sigma_{1}$ from $B$. Go to Step 1. This time the set $G \cup R$ contains $\left\{r_{0}, r_{1}, r_{3}\right\}$ which is a spanning set for $\mathbb{R} \sigma_{2}$. Therefore, remove $\sigma_{2}$ from $B$ and place $r_{6}$ in $R$. Any support function $h$ is completely determined by its values on $r_{1}, r_{2}, r_{4}$ and $r_{5}$, so $\kappa_{0} \leq 4$.

Example 4.6. Let $\Delta$ be a complete fan on $\mathbb{R}^{3}$ and assume that the intersection of the fan $\Delta$ with the unit sphere $S$ traces a graph that corresponds to the edges of a cube as shown in Figure 2(b). Applying Algorithm 4.4 to $\Delta$, we see that $\rho_{1}(\Delta) \leq 1$ and $\rho_{2}(\Delta) \leq 1$.

Take $\Delta(1)$ to be $\left\{\mathbb{R}_{\geq} \eta_{i} \mid i=0 . .7\right\}$ where $\left\{\eta_{0}, \ldots, \eta_{7}\right\}=$

$$
\left\{\left(\begin{array}{l}
1 \\
1 \\
1
\end{array}\right),\left(\begin{array}{c}
1 \\
-1 \\
1
\end{array}\right),\left(\begin{array}{c}
-1 \\
-1 \\
1
\end{array}\right),\left(\begin{array}{c}
-1 \\
1 \\
1
\end{array}\right),\left(\begin{array}{c}
1 \\
1 \\
-1
\end{array}\right),\left(\begin{array}{c}
1 \\
-1 \\
-1
\end{array}\right),\left(\begin{array}{c}
-1 \\
-1 \\
-1
\end{array}\right),\left(\begin{array}{c}
-1 \\
1 \\
-1
\end{array}\right)\right\} .
$$


Using the methods of [5, Section 4] we find that the upper bounds predicted by Algorithm 4.4 are reached: $\rho_{1}(\Delta)=1$ and $\rho_{2}(\Delta)=2$.

Now change the fan so that $\Delta^{\prime}(1)$ is no longer symmetrical about the origin. For example, take $\Delta^{\prime}(1)$ to be $\left\{\mathbb{R}_{\geq} \eta_{i} \mid i=0 . .7\right\}$ where $\left\{\eta_{0}, \ldots, \eta_{7}\right\}=$

$$
\left\{\left(\begin{array}{l}
2 \\
1 \\
1
\end{array}\right),\left(\begin{array}{c}
1 \\
-1 \\
1
\end{array}\right),\left(\begin{array}{c}
-1 \\
-1 \\
1
\end{array}\right),\left(\begin{array}{c}
-1 \\
1 \\
1
\end{array}\right),\left(\begin{array}{c}
1 \\
1 \\
-1
\end{array}\right),\left(\begin{array}{c}
1 \\
-1 \\
-1
\end{array}\right),\left(\begin{array}{l}
-1 \\
-1 \\
-1
\end{array}\right),\left(\begin{array}{c}
-1 \\
1 \\
-1
\end{array}\right)\right\} .
$$

Using the methods of [5, Section 4] we find that the lower bounds predicted by Conjecture 4.3 are attained: $\rho_{1}\left(\Delta^{\prime}\right)=0$ and $\rho_{2}\left(\Delta^{\prime}\right)=1$. Now Pic $X^{\prime}$ is torsion-free for the complete toric variety $X^{\prime}=T_{N}$ emb $\Delta^{\prime}$. Since $\rho_{1}\left(\Delta^{\prime}\right)=0$ we see that $\operatorname{Pic} X^{\prime}=0$. This proves that $X^{\prime}$ is nonprojective (see Remark 7). If $B$ is an open neighborhood of $\Delta$, then the strata of $B$ are $\kappa_{0}=4$ and $\kappa_{0}=3$. We will show later that $\kappa_{0}=4$ corresponds to a Zariski closed subset of $B$.

Remark 7. In general any toric variety satisfying Conjecture 4.3 is nonprojective. This is because a projective normal variety $X$ will always have a nonprincipal Cartier divisor corresponding to a hyperplane section. This follows from commutative diagram (29). See [8, Ex. 6.2, p. 146].

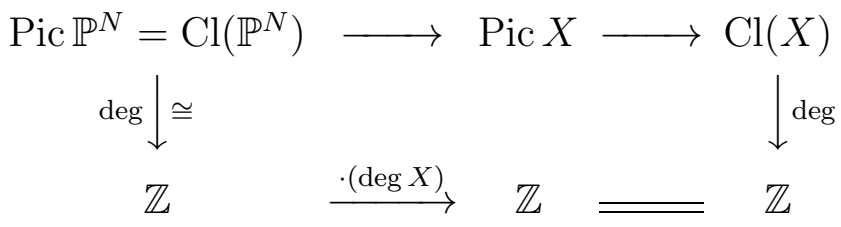

Example 4.7. We give an example to illustrate how (27) can be used to compute $\kappa_{0}$. Say $\Delta$ consists of three 3-dimensional cones as shown in Figure $\mathbb{2}\left(\right.$ a). Then $\mathcal{W}\left(\Delta_{\text {top }}\right)=\mathbb{Z} r_{0} \oplus \cdots \oplus \mathbb{Z} r_{6}$ and $\mathcal{W}\left(\Delta\left(\sigma_{0}\right)\right)=\mathbb{Z} r_{0} \oplus \mathbb{Z} r_{1} \oplus \mathbb{Z} r_{2} \oplus \mathbb{Z} r_{4}$. Let $\eta_{i}$ be a primitive lattice point on $r_{i}$, so that $r_{i}=$ $\mathbb{R}_{\geq} \eta_{i}$ for $i=0 . .6$. The kernel of the surjection $\phi_{0}: \mathcal{W}(\Delta) \rightarrow \mathrm{Cl}\left(U_{\sigma_{0}}\right)$ is spanned by the vectors $\left(\begin{array}{lllllll}0 & 0 & 0 & 1 & 0 & 0 & 0\end{array}\right)^{\top},\left(\begin{array}{lllllll}0 & 0 & 0 & 0 & 0 & 1 & 0\end{array}\right)^{\top},\left(\begin{array}{lllllll}0 & 0 & 0 & 0 & 0 & 0 & 1\end{array}\right)^{\top}$, and the columns of $\left(\begin{array}{lllllll}\eta_{0} & \eta_{1} & \eta_{2} & 0 & \eta_{4} & 0 & 0\end{array}\right)^{\top}$. So ker $\phi_{0}$ is a subspace of codimension 1. Consider the matrix equation

$$
\left(\begin{array}{llll}
\eta_{0} & \eta_{1} & \eta_{2} & \eta_{4}
\end{array}\right) \overrightarrow{v_{0}}=0
$$

Set $A=\left(\begin{array}{lll}\eta_{0} & \eta_{1} & \eta_{2}\end{array}\right)$. Then (30) becomes

$$
\left(\begin{array}{ll}
I & A^{-1} \eta_{4}
\end{array}\right) \overrightarrow{v_{0}}=0 .
$$

Set $A^{-1} \eta_{4}=\left(\begin{array}{lll}a_{0} & b_{0} & c_{0}\end{array}\right)^{\top}$. So $\overrightarrow{v_{0}}=\left(\begin{array}{llll}-a_{0} z & -b_{0} z & -c_{0} z & z\end{array}\right)^{\top}$. Since any 3 columns of the matrix in (30) are linearly independent, $\overrightarrow{v_{0}}$ has 4 nonzero entries, or $\overrightarrow{v_{0}}=0$. Normalize $\overrightarrow{v_{0}}$ by taking $z=-1$. Then $\operatorname{ker} \phi_{0}$ is the set of solutions to

$$
\left(\begin{array}{lllllll}
a_{0} & b_{0} & c_{0} & 0 & -1 & 0 & 0
\end{array}\right) \vec{x}=0 .
$$

Hence if $\phi: \mathcal{W}(\Delta) \rightarrow \mathrm{Cl}\left(U_{\sigma_{0}}\right) \oplus \mathrm{Cl}\left(U_{\sigma_{1}}\right) \oplus \mathrm{Cl}\left(U_{\sigma_{2}}\right)$, then $\operatorname{ker} \phi$ is the set of solutions to

$$
\left(\begin{array}{ccccccc}
a_{0} & b_{0} & c_{0} & 0 & -1 & 0 & 0 \\
a_{1} & 0 & b_{1} & c_{1} & 0 & -1 & 0 \\
a_{2} & b_{2} & 0 & c_{2} & 0 & 0 & -1
\end{array}\right) \vec{x}=0
$$


This coefficient matrix has rank 3 so ker $\phi$ has rank 4 . Therefore $\kappa_{0}=4$ and for any open neighborhood $B$ of $\Delta, B$ has only 1 stratum. In this case, we see that $\kappa_{0}$ and hence $\rho_{1}$ and $\rho_{2}$ are topological invariants of the set of all (rational) fans that look like the one shown in Figure 2(a). We could assume $\Delta$ has more than three (say $w+1$ ) 3-dimensional cones each with four 1-dimensional faces meeting around the common 1-dimensional face $r_{0}$. By a similar argument we see that $\kappa_{0}=w+2$.

Example 4.8. Let $\Delta$ be a fan on $\mathbb{R}^{3}$ such that $\Delta_{t o p}$ is homeomorphic to the fan in Example 3.6. Following the procedure of Example 4.7, set up equations analogous to (30) (32) and (33). Then $\operatorname{ker} \phi$ is the set of solutions to

$$
\left(\begin{array}{cccccc}
a_{0} & 0 & b_{0} & c_{0} & 0 & -1 \\
a_{1} & b_{1} & 0 & c_{1} & -1 & 0 \\
0 & a_{2} & b_{2} & 0 & c_{2} & -1
\end{array}\right) \vec{x}=0 .
$$

The coefficient matrix in (34) clearly has rank 2 or more. This agrees with the upper bound 4 predicted for $\kappa_{0}$ by Algorithm 4.4. The third, fourth and sixth columns of (34) are independent if and only if

$$
\left(b_{2}-b_{0}\right) c_{1} \neq 0 \text {. }
$$

This shows that on the complement of a Zariski open subset of $B, \kappa_{0}=3$. We check that (35) is satisfied on a nonempty subset of $B$. Note that (35) is satisfied if

$$
\text { the second row of }\left(\begin{array}{llll}
\eta_{0} & \eta_{2} & \eta_{3}
\end{array}\right)^{-1} \eta_{5} \neq \text { the second row of }\left(\begin{array}{lll}
\eta_{1} & \eta_{2} & \eta_{4}
\end{array}\right)^{-1} \eta_{5}
$$

which will be true for a sufficiently general choice of the fan. To see this, consider letting $p_{0}$ vary in $B_{0}$. Then in (36) the matrix $\left(\begin{array}{llll}\eta_{0} & \eta_{2} & \eta_{3}\end{array}\right)^{-1}$ varies but the matrix $\left(\begin{array}{lll}\eta_{1} & \eta_{2} & \eta_{4}\end{array}\right)^{-1}$ remains constant.

Consider (27) once again. Let $\Delta$ be a complete fan on $\mathbb{R}^{3}$. Let $B$ be an open neighborhood of $\Delta$. Proceed as in Examples 4.7 and 4.8. Set up the matrix equation $\Phi \vec{x}=0$ for ker $\phi$. Since $M \rightarrow \mathrm{SF}\left(\Delta_{\text {top }}^{\prime}\right)$ is injective, ker $\phi$ has rank at least 3. Consider an arbitrary $(n-2)-$ by $-(n-2)$ submatrix $\Phi_{0}$ of $\Phi$. Then $\Phi_{0}$ has rank $n-2$ exactly when $\operatorname{det}\left(\Phi_{0}\right) \neq 0$. As in (34) and (35), we can show that $\operatorname{det}\left(\Phi_{0}\right)=0$ is an equation in no more than $3(n-2)$ variables which are parametrized by points in $B$. The equation $\operatorname{det}\left(\Phi_{0}\right)=0$ defines a Zariski closed subset of $B$. On the complement of this closed set $\operatorname{det}\left(\Phi_{0}\right) \neq 0, \operatorname{rank} \Phi=n-2$ and ker $\phi$ has $\operatorname{rank}$ 3. If there is at least one choice of $\Delta_{\text {fan }}$ for which $\operatorname{det}\left(\Phi_{0}\right) \neq 0$, then the open set making up the complement of the determinant equations will be nonempty, hence the conclusion of Conjecture 4.3 will be satisfied. This shows for example that the general fan which is topologically homeomorphic to that of Figure 2(b) satisfies the conclusion to Conjecture 4.3, because in Example 4.6 an example is given which shows the determinants are nonzero on a nonempty Zariski open in $B$.

\section{REFERENCES}

[1] L. Childs, On Brauer groups of some normal local rings, in Brauer Groups, Evanston 1975, vol. 549 of Lecture Notes in Mathematics, 1976, Springer-Verlag, Berlin, pp. 1-15.

[2] V. I. Danilov, The geometry of toric varieties, Russian Math. Surveys, 33 (1978), pp. 97-154. (Uspekhi Mat. Nauk, 33:2 (1978), pp. 85-134).

[3] F. R. DeMeyer and T. J. Ford, On the Brauer group of toric varieties, Trans. Amer. Math. Soc., 335 (1993), pp. 559-577. 
[4] F. R. DeMeyer, T. J. Ford, and R. Miranda, The cohomological Brauer group of a toric variety, J. of Alg. Geom., 2 (1993), pp. 137-154.

[5] T. J. Ford, Examples of locally trivial Azumaya algebras, in 1992 Summer Reseach Institute on Quadratic Forms and Division Algebras, W. Jacob and A. Rosenberg, eds., Proceedings of Symposia in Pure Mathematics, Amer. Math. Soc., Providence, to appear.

[6] W. Fulton, Introduction to Toric Varieties, vol. 131 of Annals of Mathematics Studies, Princeton University Press, Princeton, New Jersey, 1993.

[7] A. Grothendieck, Le groupe de Brauer I, II, III, in Dix Exposés sur la Cohomologie des Schémas, North Holland, Amsterdam, 1968, pp. 46-188.

[8] R. Hartshorne, Algebraic Geometry, vol. 52 of Graduate Texts in Mathematics, Springer-Verlag, New York/Berlin, 1977.

[9] R. Hoobler, Functors of graded rings, in Methods in Ring Theory, Antwerp, 1983, F. van Oystaeyen, ed., NATO ASI Series, Reidel, Dordrecht, 1984, pp. 161-170.

[10] T. Oda, Convex Bodies and Algebraic Geometry, vol. 15 of Ergebnisse der Mathematik und ihrer Grenzgebiete, 3. Folge, Springer-Verlag, Berlin/Heidelberg, 1988.

Department of Mathematics, Florida Atlantic University, Boca Raton, Florida 33431

E-mail address: Ford@@acc.fau.edu 